\title{
Qualitative analysis of remineralized carious lesions subjected to fluoride supplement through confocal laser scanning microscope
}

\author{
K. Shashikala*, N. V. Sheela
}

Department of Conservative Dentistry and Endodontics, R. V. Dental College\& Hospital, Bangalore, India. Email: shashbang2007@rediffmail.com

Received 10 June 2011; revised 25 July 2011; accepted 3 August 2011.

\section{ABSTRACT}

Aim: 1] Comparative evaluation of the linear depth of induced remineralized lesions after subjecting to fluoride supplements and 2] To assess the average fluorescence at both the demineralized and the remineralized zones in all the three study groups under confocal laser scanning microscope. Method: Forty five sound human premolars extracted for orthodontic reasons were decoronated $1 \mathrm{~mm}$ below the cemento-enamel junction and coated with nail varnish except for a $3 \times 3 \mathrm{~mm}$ window on the buccal surface. The samples were placed in $50 \mathrm{ml}$ of de mineralizing solution at pH 4.6 for 96 hours. Following demineralization, the lower half of the $3 \times 3 \mathrm{~mm}$ window in all the samples were covered with nail varnish to serve as control. The samples were randomly divided into three groups of fifteen teeth each $(n=15)$ and specimens in group $A[\mathrm{Nfd}]$ were remineralized using non-fluoridated dentifrice [control], those in groups B [Fd5] and group C [Fd10] using 500 ppm and 1000 ppm of fluoride containing dentifrice, respectively. The specimens were subjected to a 20 day remineralization treatment regimen and were sectioned into $100 \mu \mathrm{m}$ thick sections and two images were captured on the buccal surface from either side of the midpoint of occluso-cervical length using confocal laser scanning microscope [CLSM]. Results: were tabulated and statistically analyzed by Anova. Study concluded that 1000 ppm fluoridated dentifrice showed a greater degree of remineralization than other groups and confocal laser scanning microscopes gives promising results in the diagnosis of early enamel lesions over the conventional methods.

Keywords: Dental Caries; Fluoridated Dentifrice; Confocal Laser Scanning Microscope

\section{INTRODUCTION}

Dental caries is a complex disease process that afflicts a large population of the world, regardless of gender, age and ethnicity, although it does tend to affect more individuals with a low socio-economic status to a greater extent. Tooth minerals are lost and regained constantly in the human oral environment. The tooth health is hence dependent upon equilibrium of this mineral exchange. A break in the equilibrium causes the tooth to either demineralize or remineralize depending upon the concentration of the mineral saturation in the oral cavity [1].

Over the last few decades, fluoride in various forms has been proven to reduce caries in both the primary and permanent dentitions when used in a variety of ways. This is because it acts as a catalyst and influences reaction rates with dissolution and transformation of hydroxyapatite to fluorapatite that resists the demineralization process in the tooth. Various topical agents like fluoridated solutions, gels, mouth rinses and dentifrices have been used to hasten the remineralization of these carious lesions [2]. Fluoridated dentifrice in various concentrations has been used to bring about remineralization of the carious lesion since it is a most common and easily available vehicle that is used to cleanse the teeth worldwide and can deliver fluoride topically to the oral cavity. Remineralization of the carious lesions caused by the action of fluoride supplements have been analyzed by various qualitative and quantative techniques of measurement of tooth mineral changes that include Polarized Light Microscopy (PLM), light scattering, Polarization-Sensitive Optical Coherence Tomography, Transverse Microradiography, Energy Dispersive X-ray analysis, cross-sectional microhardness determination and Confocal Laser Scanning Microscopy. Confocal laser scanning microscopy is a powerful imaging technique based upon optical behavior of light within specimens. Generally, confocal analysis features excita- 
tion (such as fluorescence) although emission detection is also possible [3]. The optical property of this technique helps in the detection of carious and non-carious lesions of the tooth [4]. To date, not much information is available on the usage of confocal microscope for the analysis of early enamel lesions of the tooth.

Therefore, the aim of the present study is 1) Comparative evaluation of the linear depth of induced remineralized lesions [measured in microns] after subjecting to fluoride supplements and 2) To assess the average fluorescence at both the demineralized and the remineralized zones in all the three study groups under confocal laser scanning microscope.

\section{MATERIALS AND METHODS}

Forty five healthy human premolars which were extracted for orthodontic purposes and with no patient identifiers were collected from the Department of Oral and maxillofacial Surgery. The teeth were cleaned thoroughly to remove any blood, soft tissue or extrinsic deposits with hand scaler. They were stored in $100 \mathrm{ml}$ of $10 \%$ buffered formalin ( $\mathrm{pH} 6.8$ to 7.0 ) for a period of three months until they were used for the study. Teeth were sectioned $1 \mathrm{~mm}$ below the cemento-enamel junction with a slow speed diamond disc. The roots were discarded and the crowns were used for the study.

\subsection{Artificial Smooth-Surface Enamel Lesion Formation (Demineralization Procedure)}

All the samples were covered with nail varnish leaving a window of $3 \times 3 \mathrm{~mm}$ on the buccal surface and were kept in a $50 \mathrm{ml}$ of de mineralizing solution in a sterile glass beaker, which was a mixture of calcium chloride [2.0 $\mathrm{mmol} / \mathrm{L}]$, tri sodium phosphate $[2.0 \mathrm{mmol} / \mathrm{L}]$ in acetate buffer $[75 \mathrm{mmol} / \mathrm{L}]$ solution at $\mathrm{pH} 4.6$ for 96 hours. Following demineralization, the lower half of the $3 \times 3 \mathrm{~mm}$ window in all the samples were covered with nail varnish to serve as control. The samples were randomly divided into three groups of fifteen teeth each [n =15] before they were being subjected to the $\mathrm{pH}$ cycle.

Group Nfd: Remineralization of the lesion using nonfluoridated dentifrice tooth paste [Meswak Dabur, India] was used as a control.

Group Fd5: Remineralization of the lesion using 500 ppm of fluoride containing Dentifrice Bubble Fruit tooth paste [Colgate, India].

Group Fd10: Remineralization of the lesion using 1000 ppm of fluoride containing Dentifrice-Colgate Total tooth paste [Colgate, India].

\subsection{Stimulated Whole Saliva Collection}

Five healthy female adult volunteers in the age group of 18 yrs - 25 yrs who had full complement of teeth with no past caries experience were chosen for the collection of saliva between 9 am to 11 am, after obtaining clearance from institutional ethical committee and written informed consents from participating individuals. The selected subjects were not on any medication that influenced the salivary flow rate or had any salivary pathology, with no faulty dental restorations, no current caries activity, and no active periodontal disease, and were neither with dental prosthesis nor with orthodontic therapy. They were made to brush the teeth with a nonfluoridated dentifrice and later made to rinse thoroughly prior to the collection of saliva. They were then made to chew on paraffin wax and empty the stimulated saliva into sterile containers in about 5 mins. The $15 \mathrm{ml}$ of the sample was collected and stored under refrigeration until use.

\subsection{The Ph Cycling Regimen (Remineralization Procedure)}

Group Nfd: Sample of 15 teeth were subjected to a 20 day remineralization treatment regimen by immersion in fresh non-fluoridated dentifrice slurry prepared by adding $5 \mathrm{~g}$ of dentifrice in $10 \mathrm{ml}$ of water in a glass beaker for 2 mins, followed by rinsing with distilled water. The remineralizing period wherein the teeth were immersed in $15 \mathrm{ml}$ of collected saliva sample was for $2 \mathrm{hrs}$.

The teeth were then subjected to 4 hrs of acid challenge in order to simulate the oral environment like the in-vivo conditions, where samples were subjected to 15 $\mathrm{ml}$ of demineralizing solution, followed by 2 hrs of immersion in $15 \mathrm{ml}$ of saliva and then subjecting it to the dentifrice for 2 mins again and then immersion in saliva for the rest 16 hrs. The saliva samples were changed each day during the acid challenge period. The procedure was repeated for Groups Fd5 and Fd10 specimens, wherein the dentifrices used contained 500 and 1000 ppm of fluoride respectively, unlike the non-fluoridated dentifrice used in Group NFd.

\subsection{Post-Treatment Analysis}

Following the treatment period the specimens were mounted in self-cure acrylic resin. The samples were sectioned longitudinally perpendicular to the varnished area so that each section included the varnish-covered baseline lesion area and the uncovered, post-treatment lesion area with a hard tissue microtome (Leica SP 1600) to obtain specimens that were 100 microns thick.

The sections were polished with an abrasive stone and were then stained with freshly prepared $0.1 \mathrm{mM}$ Rhodamine B solution for $1 \mathrm{hr}$. The stained samples were washed thoroughly with phosphate buffer solution until there was no dye leaching out of the sample. All samples were mounted on frosted glass slides with $80 \%$ glycerol mountant and the edges of the cover slip were coated 
with transparent nail enamel for further analysis through the Confocal Laser Scanning Microscope, Leica TCS SL inverted microscope.

The images with CLSM were captured from the buccal surface that is one each from either side of the midpoint measured from the occluso-cervical length of the tooth at $[5 \times]$ magnification and Argon laser was used at $488 \mathrm{~nm}$ wavelength for excitation and emission range of 498 - $514 \mathrm{~nm}$ wavelength. Two images were captured on the buccal surface from either side of the midpoint of occluso-cervical length. The buccal surface areas were scanned between 10 and 50 microns below the cut surface and the superficial areas were not scanned because of the concern regarding the smear layer. The captured images were calibrated for linear depth of fluorescence and also the average/total fluorescence of the lesion using the Leica TCS SL in-built software, Germany.

\section{RESULTS}

A Comparative evaluation of all the samples was undertaken to study, 1) The linear depth of lesions in microns $[\mu \mathrm{m}]$, as seen through con focal laser scanning microscope and 2) To assess the average fluorescence at both the demineralized and the remineralized zones in all the three study groups under confocal laser scanning microscope.

The induced carious lesion depths were assessed under CLSM by measuring from the enamel surface to the deeper zone of the lesion and an average of ten measurements were taken from every image that was captured both in the demineralized and remineralized zone of the $3 \times 3 \mathrm{~mm}$ window of every specimen of all the three groups. The measurements were tabulated and statistically analyzed by Anova and student t test (Tables 1 and 2).
The extent of $0.01 \mathrm{mM}$ Rhodamine B dye penetration seen as fluorescence in the specimens under Argon Laser were also calibrated and recorded with the in-built software [Leica TCS SL, Germany]. The average of the mean amplitude obtained from the images of the specimens of the three groups was also tabulated and analyzed (Figures 2 and 3).

Confocal microscopic evaluation in the present study shows that the highest value for demineralization after 96 hrs was seen in the control group, which was 183.41 $\pm 7.81 \mu \mathrm{m}$ and the lowest was for group NF5 [500 ppm dentifrice] which was $180.91 \pm 7.78 \mu \mathrm{m}$. Similarly the highest value of remineralization was $156.74 \pm 7.95 \mu \mathrm{m}$ seen in the group NFd specimens showing that the values were significant $[p<0.001]$ and the remineralization values were $124.07 \pm 3.46 \mu \mathrm{m}$ lowest for group Fd10 [1000 ppm dentifrice]. A p-value of 0.616 was obtained for the demineralization under CLSM (Figure 1).

Descriptive statistical analysis was carried out in the present study. Results on continuous measurements were presented on Mean \pm SD (Min-Max) and results on categorical measurements are presented in Number (\%). Significance was assessed at 5\% level of significance. Analysis of variance (ANOVA) was used to find the significance of study parameters between three or more groups of specimens, Student $t$ test (two tailed, independent) was used to find the significance of study parameters on continuous scale between two groups (Inter group analysis) on metric parameters, Student t test (two tailed, dependent) was used to find the significance of study parameters on continuous scale within each group.

\section{DISCUSSION}

The use of artificial caries systems has greatly increased

Table 1. Evaluation of linear depth of lesion in $\mu \mathrm{m}$ as seen through confocal laser scanning microscope for both demineralized and remineralized areas.

\begin{tabular}{ccccc}
\hline Zone & Group NFd [Control] & Group Fd5 & Group Fd10 & P value \\
\hline Demineralised & $183.41 \pm 7.81$ & $180.91 \pm 7.78$ & $181.54 \pm 5.84$ & 0.616 \\
Remineralized & $156.74 \pm 7.95$ & $144.65 \pm 5.71$ & $124.07 \pm 3.46$ & $<0.001^{* *}$ \\
Difference & $26.67 \pm 13.01$ & $36.26 \pm 7.57$ & $57.47 \pm 6.67$ & - \\
P value & $<0.001^{* *}$ & $<0.001^{* *}$ & $<0.001^{* *}$ & - \\
\hline
\end{tabular}

Table 2. Evaluation of average fluorescence in $\mu \mathrm{m}$ under Con focal laser scanning microscope.

\begin{tabular}{ccccc}
\hline Zone & Group Nfd [Control] & Group Fd5 & Group Fd10 & P value \\
\hline Demineralized & $83.41 \pm 7.81$ & $80.31 \pm 8.95$ & $80.07 \pm 8.41$ & 0.363 \\
Remineralized & $54.07 \pm 6.44$ & $40.29 \pm 4.77$ & $25.54 \pm 4.63$ & $<.0014^{* *}$ \\
Difference & $29.34 \pm 9.13$ & $40.03 \pm 7.87$ & $54.53 \pm 9.73$ & - \\
P value & $<0.001^{* *}$ & $<0.001^{* *}$ & $<0.001^{* *}$ \\
\hline
\end{tabular}

+ Suggestive significance (P value: $0.05<\mathrm{P}<0.10$ ); * Moderately significant (P value: $0.01<\mathrm{P} \leq 0.05) * *$ strongly significant $(\mathrm{P}$ value: $\mathrm{P} \leq 0.01)$. 


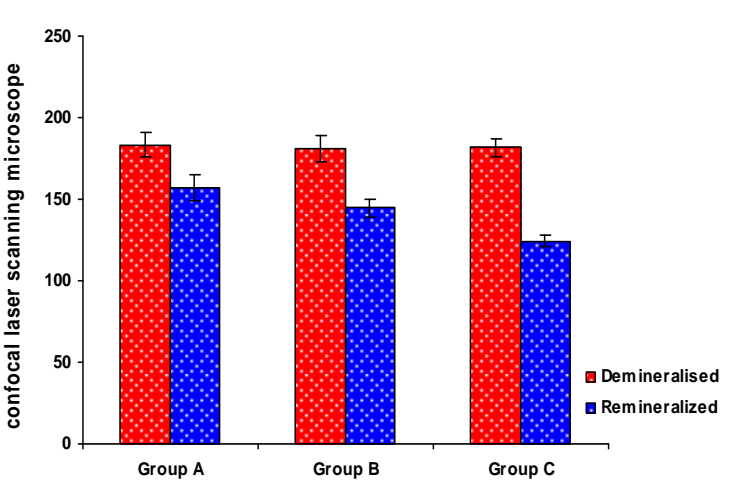

(a)

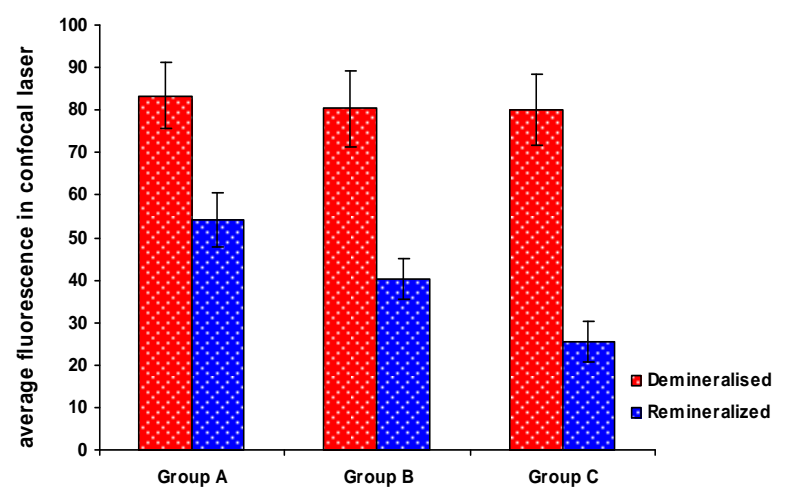

(b)

Figure 1. (a) Average linear depth of lesion in $\mu \mathrm{m}$ as seen through confocal laser scanning microscope for both de mineralized and re mineralized areas. (b) Average fluorescence in $\mu \mathrm{m}$ as seen through the confocal laser scanning microscope for both de mineralized and re mineralized areas.

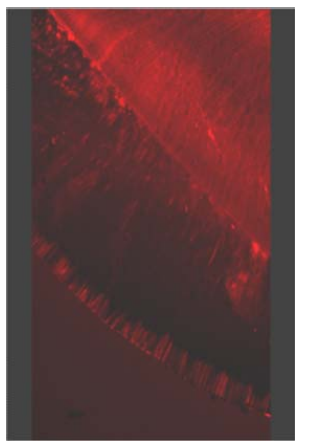

(a)

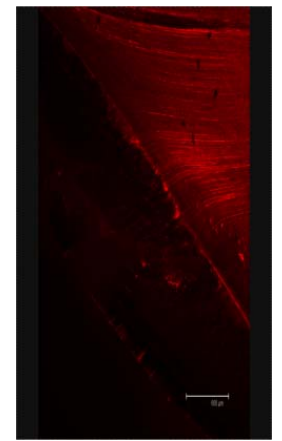

(b)

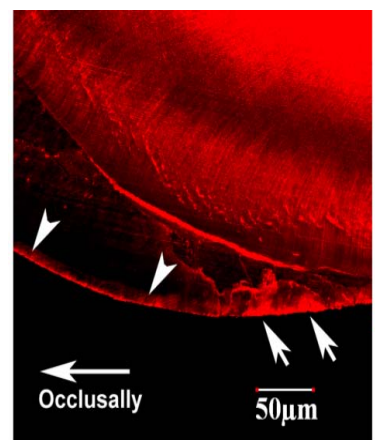

(c)

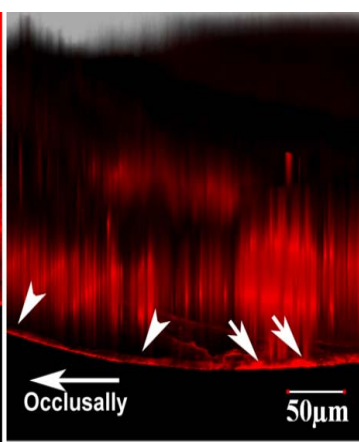

(d)

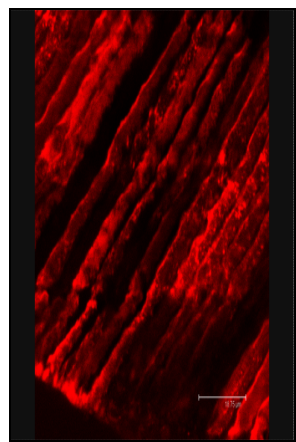

(e)

Figure 2. CLSM images [5× $]$ of occlusal aspect of the $3 \times 3 \mathrm{~mm}$ window of specimens. (a) Nfd group.(b) Fd5 group (c) Fd10 group showing $2 \mathrm{D}$ and (d) $3 \mathrm{D}$ reconstruction of the images at every $10 \mu$ stack, arrows indicate de mineralized and arrow heads indicate re mineralized zones. (e) Image of group Fd10 specimen $[20 \times$ zoom] showing demineralized and re mineralized zones and the enamel rods.

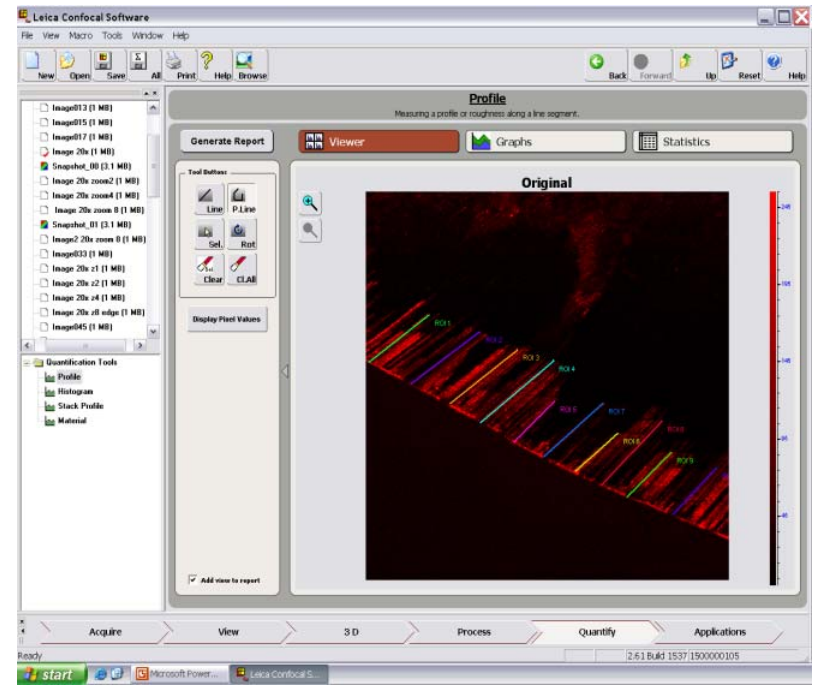

(a)

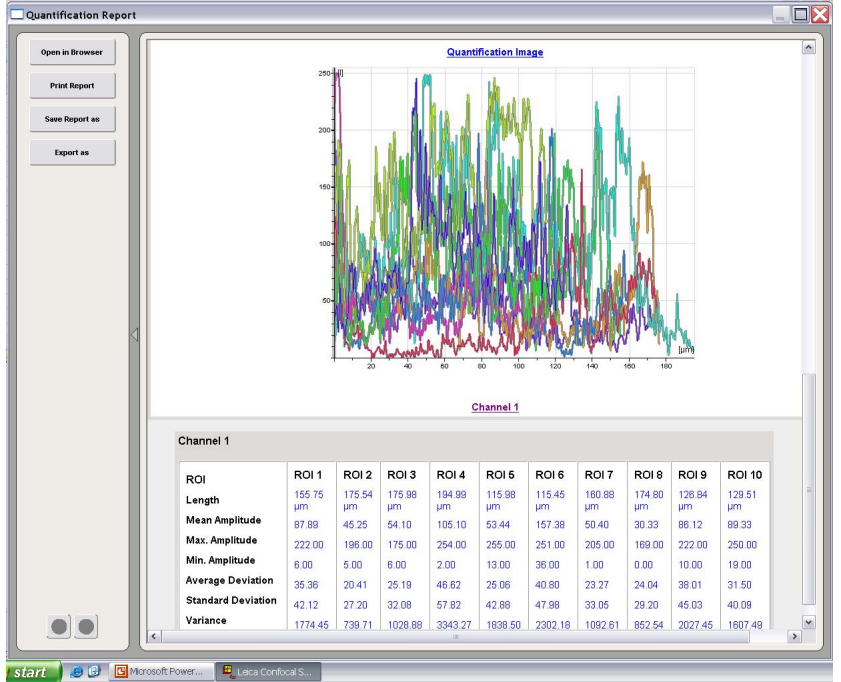

(b)

Figure 3. (a) Images showing the method of calibration with the Leica TCS SL in-built software. (b) Calibrated Image showing the graph of the intensity of fluorescence along the ten measurements (ROI 1-10) and the table showing the length measurements and means amplitude along with minimum and maximum amplitude of fluorescence. 
our knowledge of the demineralization and remineralization process [13]. The administration of fluoride has been proposed, and used, as a method of reducing enamel susceptibility to decalcification since the time it was discovered in the early 20th century by eminent investigators like McKay and Black [5]. Fluoride affects the caries process by enabling the formation of high quality fluorapatite that aids remineralization and inhibits glycolysis of plaque microorganisms [6]. The early attempts to incorporate fluoride into toothpastes which dates back to the 1960s and it is one of the most common and effective modes of topical fluoride delivery [7].

Hence, in the present study a non-fluoridated dentifrice has been used as a control along with two higher concentrations of fluoride, that is 500 ppm and 1000 ppm for comparison. The study was designed in such a way that it simulated an oral environment subjected to acid and remineralization twice a day.

Monitoring demineralization and remineralization of dental hard tissues is essential for the prevention and minimally invasive treatment of dental caries and hypersensitivity [8]. Light based methods used for the detection of dental caries induced enamel demineralization are Conventional radiography, micro radiography, Digital Imaging Fiber Optic Trans illumination Imaging [DIFOTI], laser induced fluorescence [DIAGNO dent], and quantitative light-induced fluorescence [QLF]. Conventional microscopy suffers from the problem as light scattering, namely multiple scattering from objects that are out of focus within the illuminated region prevents imaging deep within a sample. Further, if care is not taken, optical microscopy can lead to the observation of certain artifacts which in turn leads to incorrect physical interpretation of the system in question [3,9].

Confocal microscopy and its application in dentistry have been advocated by Watson [9]. CLSM overcomes the limitations of conventional wide-field microscopy through: high resolution fluorescence, high resolution 3-D imaging, non-destructive imaging technique and in-situ characterization of tissue microstructure images. Fontana performed a study to correlate area of lesion, average fluorescence and total fluorescence obtained by con focal microscopy to lesion depth and mineral loss obtained from micro radiography and polarized light microscopy. The findings show that when a $0.1 \mathrm{mM}$ solution of rhodamine $\mathrm{B}$ dye was used, the lesion area correlated well with the mineral loss obtained from micro radiography [10].

However, the average lesion fluorescence best represented mineral loss, based on their hypothesis that rhodamine $\mathrm{B}$ penetrates the voids and pores created during enamel demineralization which is a better method of evaluation over the birefringence through CLSM. Ca- bezas also conducted a correlation study between micro radiography and con focal microscopy for measurement of enamel remineralization. As described by Fontana, 0.1 $\mathrm{mM}$ rhodamine $\mathrm{B}$ dye was used and lesion area, total fluorescence and average fluorescence were evaluated. Lesion area was the confocal parameter that showed the strongest correlation with microradiography, followed by total lesion fluorescence $[8,10]$.

The present study showed a lesser degree of remineralization with the non-fluoridated dentifrices which was similar to the study by Gonzalez [10], who had used 250 ppm and 1100 ppm dentifrices along with a placebo control. It was also seen in the study that the linear depth measurements of the lesion both in the de mineralized zone and the re mineralized zone varied significantly under the con focal microscopic. This is on par with the analysis by the earlier studies [11,12] showing that con focal microscopy is an advanced tool to diagnose and measure early enamel lesions both in-vivo and in-vitro without requiring the tooth to be sectioned.

Fluorides are effective means of remineralization of carious lesions in higher concentrations (1000 ppm) as compared to the non-fluoridated control or 500 ppm concentration [13]. Diagnosis of early enamel carious lesions still remains a challenge to dentists worldwide. Various in-vitro studies show promising results with con focal laser scanning microscope as a diagnostic aid for early carious lesion detection. This approach will enable the operator to circumvent errors due to variation in the thickness of enamel at different locations of the crown. Recent evolution in techniques and technologies have facilitated a relatively wide spread adoption of this imaging diagnostic modality with increased "user friendliness and flexibility". Study concluded that 1000 ppm fluoridated dentifrice showed a greater degree of remineralization than other groups and con focal laser scanning microscopes gives promising results in the diagnosis of early enamel lesions over the conventional methods.

\section{REFERENCES}

[1] Sturdevant, C.M., Roberson, T.M. and Heymann, H.O. (2006) The art and science of operative dentistry. 5th Edition, Mosby Publishers, St. Louis.

[2] Queiroz, C.S. and Hara, A.T. (2008) PH-cycling models to evaluate the effect of low fluoride dentifrice on enamel de- and remineralization. Brazilian Dental Journal, 19, 21-27. doi:10.1590/S0103-64402008000100004

[3] Kishen, A., Shrestha, A. and Rafique, A. (2008) Fiber optic backscatter spectroscopic sensor to monitor enamel demineralization and remineralization in vitro. Journal of Conservative Dentistry, 11, 63-70.

doi:10.4103/0972-0707.44053

[4] Ferriera-Zandona, A.G., Analoui, M. and Beiswanger, 
B.B. (1998) An in vitro comparison between laser fluorescence and visual examination for detection of demineralization in occlusal pits and fissures. Caries Research, 32, 210-218. doi:10.1159/000016455

[5] Hicks, J., Garcia-Godoy, F. and Flaitz, C. (2004) Biological factors in dental caries: Role of saliva and dental plaque in the dynamic process of demineralization and remineralisation (Part II). Journal of Clinical Pediatric Dentistry, 28, 119-124.

[6] Kumar, V.L.N., Itthagarun, A. and King, N.M. (2008) The effect of casein phosphopeptide-amorphous calcium phosphate on the remineralization of artificial caries-like lesions: An in vitro study. Australian Dental Journal, 53, 34-40. doi:10.1111/j.1834-7819.2007.00006.x

[7] Zaura, E., ten Cate, J.M. (2004) Dental plaque as a biofilm: A pilot study of the effects of nutrients on plaque $\mathrm{pH}$ and dentin demineralization. Caries Research, 38, 9-15. doi:10.1159/000074357

[8] Fontana, M., Li, Y. and Dunipace, A.J. (1996) Measurement of enamel demineralization using microradiography and confocal microscopy. A correlation study. Caries
Research, 30, 317-325. doi:10.1159/000262337

[9] Watson, T.F. (1991) Applications of confocal scanning optical microscopy to dentistry. British Dental Journal, 171, 287-291. doi:10.1038/sj.bdj.4807695

[10] Gonzalez-Cabezas, C. and Fontana, M. (1998) Measurement of enamel remineralization using microradiography and confocal microscopy. Caries Research, 32, 385-392. doi:10.1159/000016475

[11] Hall, A.F., DeSchepper, E. and Ando, M. (1997) In vitro studies of laser fluorescence for detection and quantification of mineral loss from dental caries. Advance Dental Research, 11, 507-514. doi:10.1177/08959374970110041901

[12] Hafstrom-Bjorkman, U. (1996) Comparison of laser fluorescence and longitudinal microradiography for quantitative assessment of in vitro enamel caries. Caries $R e$ search, 26, 241-247. doi:10.1159/000261446

[13] Wefel, J.S. and Harless, J.D. (1984) Comparison of artificial white spots by microradiography and polarized light microscopy. Journal of Dental Research, 63, 1271-1275. doi:10.1177/00220345840630110301 